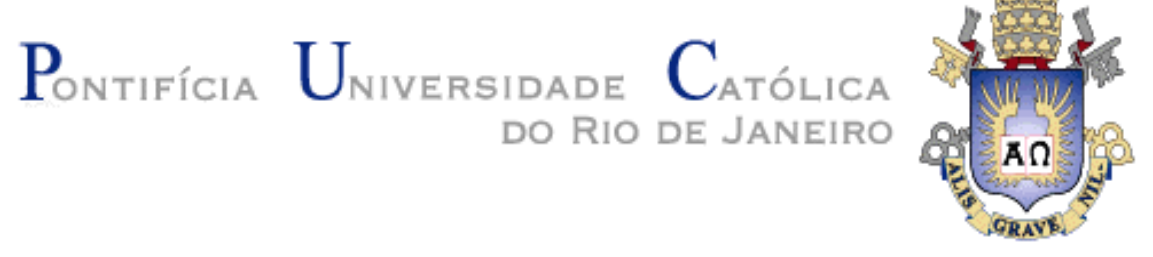

Felipe Barcelos de Aquino Ney

Matisse e a sensibilidade moderna

Dissertação de Mestrado

Dissertação apresentada como requisito parcial para obtenção do grau de Mestre pelo Programa de PósGraduação em História Social da Cultura do Departamento de História do Centro de Ciências Sociais da PUC-Rio.

Orientador: Prof. Ronaldo Brito Fernandes

Rio de Janeiro

Maio de 2010 
Felipe Barcelos de Aquino Ney

\section{Matisse e a sensibilidade moderna}

Dissertação apresentada como requisito parcial para obtenção do grau de Mestre pelo Programa de Pós-Graduação em História Social da Cultura do Departamento de História do Centro de Ciências Sociais da PUC-Rio.

Aprovada pela Comissão Examinadora abaixo assinada.

Prof. Ronaldo Brito Fernandes

Orientador

Departamento de História

PUC-Rio

\section{Prof ${ }^{\mathrm{a}}$ Patrícia Leal Azevedo Correa}

Escola de Belas Artes

UFRJ

Prof. Roberto Luís Torres Conduru

Centro de Educação e Humanidades - Instituto de Artes

UERJ

Prof ${ }^{a}$ Mônica Herz

Vice-Decano de Pós-Graduação do Centro de Ciências Sociais

PUC-Rio

Rio de Janeiro, 05 de maio de 2010. 
Todos os direitos reservados. É proibida a reprodução total ou parcial do trabalho sem autorização da universidade, do autor e do orientador.

Felipe Barcelos de Aquino Ney Graduou-se em arquitetura pela Universidade Santa Úrsula em 2003. Pós-graduado em História da Arte e Arquitetura no Brasil pela PUC-Rio em 2008

Ficha Catalográfica

Ney, Felipe Barcelos de Aquino

Matisse e a sensibilidade moderna / Felipe Barcelos de Aquino Ney ; orientador: Ronaldo Brito Fernandes. - 2010. 80 f. : il. (color.) ; $30 \mathrm{~cm}$

Dissertação (mestrado)-Pontifícia Universidade Católica do Rio de Janeiro, Departamento de História, 2010. Inclui bibliografia

1. História - Teses. 2. História social da cultura. 3. Crítica de arte. 4. Arte moderna., 5. Matisse. 6. Ingres. I. Fernandes, Ronaldo Brito. II. Pontifícia Universidade Católica do Rio de Janeiro. Departamento de História. III. Título.

CDD: 900 
Aos meus pais

Guilherme Aquino Ney, Isabel Barcelos Ney e Ana Amélia de Barcelos 


\section{Agradecimentos}

Ao meu orientador Ronaldo Brito Fernandes, por me ensinar a ver;

A João Masao Kamita, Ricardo Benzaquen e Cecília Martins de Mello pelas aulas valiosas;

À Edna Maria Timbó;

À PUC-Rio, pela parceria, estrutura e suporte;

À CAPES, pelo financiamento à minha pesquisa;

À Isabel Carneiro, pelas ricas discussões em arte;

À Baby Baptista e Mary Schneider pela força e amizade em todos os momentos;

Aos meus pais e irmãos, por tudo isso e mais um tanto. 


\section{Resumo}

Ney, Felipe Barcelos de Aquino Ney. Fernandes, Ronaldo Brito. Matisse e a sensibilidade moderna. Rio de Janeiro, 2010. 80p. Dissertação de Mestrado. Departamento de História, Pontifícia Universidade Católica do Rio de Janeiro.

Clement Greenberg anunciara há meio século que o frio hedonismo de Matisse e sua exclusão de tudo exceto a sensação concreta e imediata seria no futuro melhor compreendida como o mais profundo estado de ânimo da primeira metade do século XX. Este trabalho investiga as consequências desse hedonismo na aparência pictórica, na dupla função cartesiano-sensível, ou melhor, objetiva e subjetiva da linha elaborada por Matisse. A partir de sua manipulação sensível dos conceitos de linear e pictórico - recapitulando Wolfflin, linha e cor deixam de assumir um caráter antitético, vivem em comunhão, na condensação pela equivalência, não pela diferença, das qualidades sensíveis e intelectivas da obra. Matisse mantém os conceitos em estado de latência sensível, gerando uma espécie de percepção funâmbula de sua arte, a configurar-se como o grande vórtice da sensibilidade moderna.

\section{Palavras-chave}

Crítica de arte; arte moderna; Matisse; Ingres. 


\section{Abstract}

Ney, Felipe Barcelos de Aquino Ney. Fernandes, Ronaldo Brito (Advisor). Matisse the modern sensibility. Rio de Janeiro, 2010. 80p. MSc. Dissertation - Departamento de História, Pontifícia Universidade Católica do Rio de Janeiro.

Clement Greenberg once said that Matisse's cold hedonism and ruthless exclusion of everything but the concrete, immediate sensation will be better understood as the most profound mood of the first half of the twentieth century. The purpose of this essay is to investigate the consequences of this hedonism in the pictorial image, for to reveal how the dual sensible / intellective, objective and subjective function of the line was elaborated by Matisse. From his sentient manipulation of the pictorial and linear concepts recapitulating Heinrich Wolfflin - color and line no longer assume an anthitetical position in the pictorial image, by coexisting through equivalence, not by contrast. By maintaining the concepts in sentient latency, Matisse has generated a sort of funambulist perception of art that had become the great vortex of modern sensibility.

\section{Key words}

Art criticism; modern art; Matisse; Ingres 


\section{Sumário}

$\begin{array}{ll}\text { Introdução } & 11\end{array}$

1. Ingres e Matisse 16

1.2 Picasso e Matisse 24

2. Conceitos fundamentais da história da arte 36

3. Cézanne e Ingres 40

3.1 Cézanne e o classicismo integral 42

3.2 Os traços de Cézanne $\quad 45$

4. Objetividade e subjetividade na arte moderna 47

5. Andy Warhol 51

6. Conclusão $\quad 57$

7. Referências Bibliográficas $\quad 59$

8. Ilustrações 61 


\section{Lista de llustrações}

01. Ingres, A banhista de Valpinçon, 1808

02. Ingres, Grande odalisca, 1814

03. Matisse, "Nu rosa”, 1935

04. Ingres, Madame. Moitessier, 1856

05. Matisse, A dança, $1908 \quad 65$

06. Mondrian

Composição em vermelho, azul e amarelo, $1930 \quad 66$

07.Matisse, Lição de piano, 1916

08.William Hogarth, The country dance, 175368

09. Matisse, Mme. Matisse, $1905 \quad 69$

10.Matisse, Porta-janela em Collioure, $1914 \quad 69$

11.Matisse, Jazz (capa), $1947 \quad 70$

12. Matisse, Nu azul, $1952 \quad 71$

13.Matisse, Escargot, $1953 \quad 72$

14. Michelangelo, Biblioteca Laurenziana, 1523-1568 73

15. Matisse, Interior amarelo e azul, 1946

16.Matisse, Alegria de viver, 1906

17. Velásquez,

A infanta Margarida Tereza em vestido branco, 167376

18.Ingres, Princesa de Broglie, $1853 \quad 77$

19.Cézanne, Rapaz com colete vermelho, 1890-95 78

20.Andy Warhol, Marilyn Monroe (diptico), 1962

21.Andy Warhol, 16 Jackies, 1964 
Haud certo ergo. But Ofelicitous culpability, sweet bad cess to you for an archetypt! 